\title{
Efficient organization of nodes in wireless sensor networks (clustering location-based LEACH)
}

\author{
Mohammed Réda El Ouadi, Abderrahim Hasbi \\ Mohammadia School of Engineers, Mohammed V University, Rabat, Morocco
}

\begin{tabular}{l}
\hline \hline Article Info \\
\hline Article history: \\
Received Mar 24, 2021 \\
Revised Aug 13, 2021 \\
Accepted Aug 29, 2021 \\
\hline
\end{tabular}

Keywords:

Clustering

Energy consumption

LEACH

Network lifetime

Wireless sensor networks

\begin{abstract}
The rapid development of connected devices and wireless communication has enabled several researchers to study wireless sensor networks and propose methods and algorithms to improve their performance. Wireless sensor networks (WSN) are composed of several sensor nodes deployed to collect and transfer data to base station (BS). Sensor node is considered as the main element in this field, characterized by minimal capacities of storage, energy, and computing. In consequence of the important impact of the energy on network lifetime, several researches are interested to propose different mechanisms to minimize energy consumption. In this work, we propose a new enhancement of low-energy adaptive clustering hierarchy (LEACH) protocol, named clustering location-based LEACH (CLOCLEACH), which represents a continuity of our previous published work location-based LEACH (LOC-LEACH). The proposed protocol organizes sensor nodes into four regions, using clustering mechanism. In addition, an efficient concept is adopted to choose cluster head. CLOC-LEACH considers the energy as the principal metric to choose cluster heads and uses a gateway node to ensure the inter-cluster communication. The simulation with MATLAB shows that our contribution offers better performance than LEACH and LOC-LEACH, in terms of stability, energy consumption and network lifetime.
\end{abstract}

This is an open access article under the CC BY-SA license.

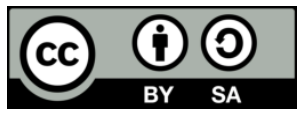

\section{Corresponding Author:}

Mohammed Réda El Ouadi

Mohammadia School of Engineers, Mohammed V University

Rabat, Morocco

Email: elouadi.mr@gmail.com

\section{INTRODUCTION}

Recently, wireless sensor networks (WSNs) have been rapid progress, through their use in different fields and domains such as military domain [1], [2], health application [3], security surveillance [4], smart home [5] and another similar area. WSNs are composed of multiple sensor nodes, which are used to collect data from a real environment, as shown in Figure 1. Sensor nodes have minimal energy, communication, and computing capabilities [6]. Generally, it is difficult to replace or change their batteries, once they are exhausted [7].

Thus, the mission to conserve the sensor nodes energy is the important challenge to be solved in WSN, due to the direct impact on the WSN lifetime [7], [8]. Random organization of nodes in the sensor area is not an efficient concept to conserve energy and provide load balancing between nodes. One of the principal methods used to organize sensor nodes is clustering, which consists of organizing nodes in groups (clusters) [9], with compliance with certain metrics. Clusters are composed of sensor nodes and one of them is elected leader and called cluster head $(\mathrm{CH})$. The $\mathrm{CH}$ is the essential element in this concept, responsible to make a special task such as: collect data from all other cluster nodes, aggregate data and transmit it directly to Base 
station (BS) or by using multi-hop [10]. Several works have offered different methods to extend lifetime of the network, by improving the factors that minimize energy consumption such as: residual energy of nodes, organization of sensor nodes in WSN, cluster head selection, routing, and data communication [11]. However, extending lifetime of the network always sufficient for many WSNs that require other metrics which depend on their domain of application and their principal deployment objective, such as, speed of data transmission or a maximum network stability period [12].

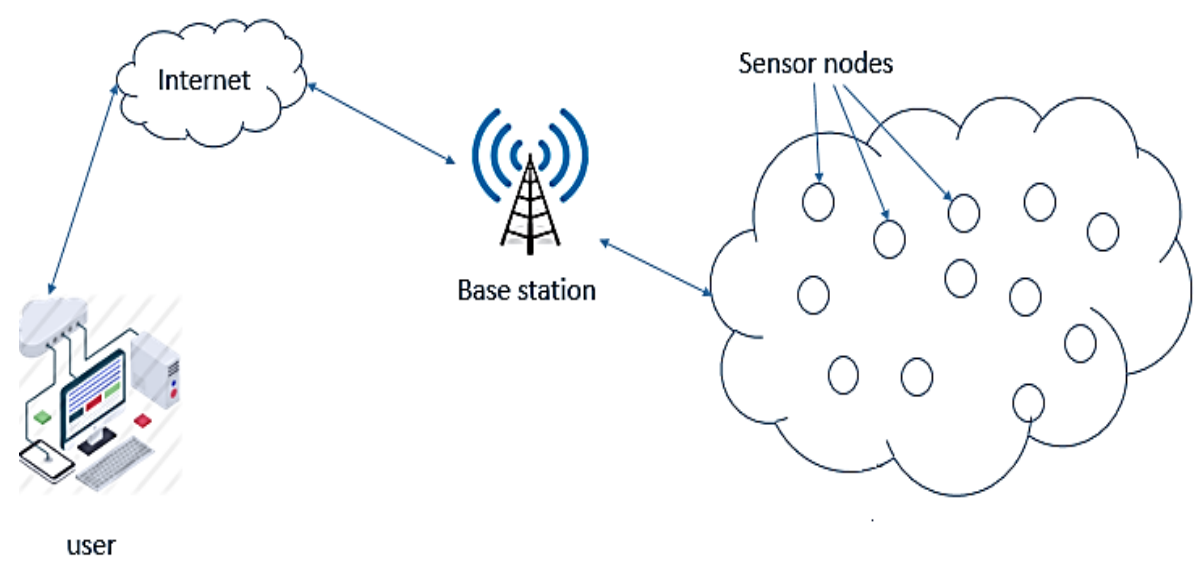

Figure 1. WSN communication architecture

This work is essentially concerned in the hierarchical routing protocols that are based on the clustering concept, and particularly low-energy adaptive clustering hierarchy (LEACH) protocol [13]. The anticipated death of nodes in $\mathrm{LEACH}$, can be solved by an efficient nodes organization in network and an efficient $\mathrm{CHs}$ election process, capable to allow a minimal energy consumption and prolong network lifetime. We propose an algorithm named clustering location based LEACH (CLOC-LEACH), that represents a new improvement of our previous contribution, location based LEACH protocol (LOC-LEACH) presented in [14].

The aim of this work is to extend the stability period of the network and enable the energy conservation of the sensor node by organizing the network into four clustered regions. Each cluster is composed of nodes belonging to the same geographic area, and an optimal $\mathrm{CH}$ election process is adopted in each of them. The $\mathrm{CHs}$ transfer their aggregated packet to gateway node according of a time division multiple access (TDMA) schedule of each $\mathrm{CH}$. Gateway node collect data from all $\mathrm{CHs}$ and transfers an aggregated packet to BS.

The remaining of the paper is structured as follows: section 2 describes the related works. While section 3 presents LEACH, LOC-LEACH protocols and describes our proposed protocol CLOC-LEACH. In section 4, performance evaluations along with comparisons are simulated and discussed. Lastly, section 5 gives conclusion.

\section{RELATED WORK}

There are multiple routing protocols that use the clustering concept to organize sensors nodes and prolong network lifetime. LEACH protocol is considered the most popular of these clustering protocols. Different protocols are proposed by several researches to improve LEACH protocol, by proposing new concepts to extend the lifetime of the WSN by minimizing the energy consumption. In 2002, an enhanced LEACH version named LEACH centralized (LEACH-C) [15] are proposed. It uses a new process of clusters formation. The $\mathrm{CHs}$ are chosen according to both principal metrics, location information and residual energy. Each sensor node transfers its information to BS. On the basis of this information, the BS defines nodes the $\mathrm{CHs}$. Each ordinary node will be assigned to its appropriate $\mathrm{CH}$. An average energy is calculated to define the CHs. The sensor node is elected $\mathrm{CH}$ only if its residual energy is less than the average energy.

Loscri et al. [16] propose two level LEACH protocol (TL-LEACH). The data is collected in each cluster as original LEACH. The principal objective is to use two levels of $\mathrm{CHs}$ when transmitting data to the $\mathrm{BS}$. The first level $\mathrm{CH}$ s that are required to transfer data to the BS are referred to as primary CHs. The second level of $\mathrm{CHs}$ is mentioned to as secondary $\mathrm{CHs}$. These $\mathrm{CHs}$ gather data from the different nodes of their 
cluster and transferring an aggregate packet to their primary $\mathrm{CH}$. A primary $\mathrm{CHs,} \mathrm{must} \mathrm{notify} \mathrm{the} \mathrm{secondary}$ $\mathrm{CHs}$ by this election and each secondary $\mathrm{CH}$ notifies its cluster members by this notification. $\mathrm{CH}$ near the base station will be used much more than $\mathrm{CH}$ far from the base station, which generates low load balancing between nodes and is the main disadvantage of this method. Vice-LEACH [17] integrates a new node status called Vice $\mathrm{CH}$, elected in each cluster. Each Vice $\mathrm{CH}$ will replace the current $\mathrm{CH}$ when its energy is exhausted. Vice-LEACH proposes this solution to solve the energy loss in LEACH during the execution of the $\mathrm{CH}$ re-election operation, by avoiding this execution in each round. Vice-LEACH allows more network lifetime but an overhead is generated due to its operation of $\mathrm{CH}$ election. Another protocol is proposed named multi-hop LEACH; the objective is to provide a better energy saving of the sensor nodes [18]. It uses the same set-up phase of $\mathrm{LEACH}$, and the $\mathrm{CHs}$ are elected themselves and other nodes are associate of the appropriate $\mathrm{CH}$. In steady state phase, each $\mathrm{CH}$ gathers data from nodes of its cluster and transfers an aggregated packet to BS. The improvement proposed by multi-hop LEACH concerns data transmission between $\mathrm{CHs}$ and $\mathrm{BS}$. Each $\mathrm{CH}$ gathers data from its cluster nodes and transfers the aggregate information to the close $\mathrm{CH}$. The protocol discovers the optimal route between each $\mathrm{CH}$ and BS through other $\mathrm{CHs}$. The $\mathrm{CHs}$ used by the optimal path represent the relay to transmit to BS.

Nguyen et al. [19] propose a new enhancement of LEACH named the Energy-LEACH protocol (E$\mathrm{LEACH}$ ). The aim objective is to enhance the $\mathrm{CH}$ selection mechanism used in LEACH. In E-LEACH the energy is considered as the key metric to select CHs. In the first round, all nodes of the network have equal amount of energy and the $\mathrm{CH}$ is chosen randomly. After the first round, the nodes with the higher energy became CHs. Hierarchical LEACH $(\mathrm{H}-\mathrm{LEACH})$ [20] minimizes the energy consumption by reducing the transmission distance during transmission phase (less distance-less power, more distance-more power). The first round is executed like LEACH. In transmission phase, master $\mathrm{CH}$ are selected to offer an optimal transmission charge by minimizing distance that separates $\mathrm{CH}$ and BS. Liu and Chinya proposes a genetic algorithm grouping system (LEACH-GA) [21]. It integrates a new preliminary step named preparation phase, before starting the steps presented by LEACH. In the preparation phase, each node performs $\mathrm{CH}$ selection process and sends message to $\mathrm{BS}$ which integrates the information of its status of being a candidate $\mathrm{CH}$ or not, identifier (ID) and its location information in the network. Then, the BS uses a genetic algorithm to select an optimal probability of nodes that will be CHs [22]. The BS diffuses a message to all nodes to advertise them about the optimal value of probability for forming clusters in the next setup phase. The preparation phase is executed once only before the first set-up phase, and the set-up and steady phases are executed like LEACH. Rodríguez et al. [6] propose an optimal clustering routing protocol based on yellow saddle goatfish algorithm (YSGA). The YSGA is responsible to configure the cluster structure and select the number of CHs. The main objective is to extend network lifetime by minimizing energy consumed by the sensor nodes.

\section{PROTOCOLS DESCRIPTION}

\subsection{LEACH protocol}

LEACH is proposed by Heinzelman et al. [13], and it is one of the principal and popular adaptive and self-organizing clustering protocols in wireless sensor networks (WSN) [22]. It proposes a randomization method, to offer a uniform energy distribution [23], [24]. LEACH algorithm is composed of two steps, namely, set-up phase, where the $\mathrm{CH}$ s are chosen and each node is assigned to its appropriate $\mathrm{CH}$ to form the cluster. The second step concerns the data to BS. It allows the social networks (SNs) to have an autonomous decision to be $\mathrm{CH}$ without control of $\mathrm{BS}$. The node decision to become a $\mathrm{CH}$ or not is related on the proportion of $\mathrm{CHs}$ for the WSN, and the number of periods when the node is elected $\mathrm{CH}$ so far. The objective is that each node becomes $\mathrm{CH}$ at least once of $\mathrm{N} / \mathrm{K}$ a round, where $\mathrm{K}$ is the count of clusters wanted and $\mathrm{N}$ represents total nodes in sensor area. Both stages of LEACH are:

a. Set-up phase: The $\mathrm{CHs}$ are chosen and other nodes choose their appropriate $\mathrm{CH}$ to form clusters. Firstly, each $\mathrm{SN}$ chooses a number between 0 and 1 . This value is confronted with T(i):

$$
\mathrm{T}(\mathrm{n})=\left(\frac{\mathrm{P}}{1-\mathrm{P} \times\left(\mathrm{r} \times \bmod \left(\frac{1}{\mathrm{P}}\right)\right)} \text { if } \mathrm{n} \in \in^{\prime \prime} G^{\prime \prime}\right.
$$

0 Elseif

$\mathrm{R}$ represents the current round; $\mathrm{P}$ represents the proportion of $\mathrm{CHs}$ defined in the WSN. G is the group of $\mathrm{CH}$ no elected yet during the last $1 / \mathrm{P}$ periods. The node can become $\mathrm{CH}$ once is value is below the threshold, else it is still an ordinary node. Once this step is achieved, each ordinary node chooses its $\mathrm{CH}$ to join its cluster. The choice of $\mathrm{CH}$ depending on received signal strength and without any consideration of the energy [25]. 
b. Transmission phase: TDMA schedule is created by each $\mathrm{CH}$ after forming cluster and diffused of their cluster members. Each $\mathrm{CH}$ gathers the data of all cluster nodes, and transfers an aggregated packet to the $\mathrm{BS}$, which contains the fused data of its cluster.

\subsection{Location based LEACH (LOC-LEACH)}

It represents an improvement of LEACH protocol, to extend the WSN lifetime and principally the period of stability [14]. In LEACH protocol, all nodes have the same possibility to be $\mathrm{CH}$, without considering their amount of energy. The $\mathrm{CH}$ performs tasks of collect, fusion and transmit the data collected to BS which quickly depletes their energy. LOC-LEACH proposes two techniques to enhance LEACH performances concerning energy conservation, lifetime, and period stability of WSN. This, by organizing the nodes of the network into three logical regions according of their location information. The nodes nearest of BS form the first region communicate directly with BS, due to the small distance between these nodes and BS. The other nodes are divided into two clustered regions. LOC-LEACH adopts a new $\mathrm{CH}$ election process, considering node energy as the main metric to select CHs. The protocol is structured in four phases:

a. Initialization phase: In this phase, the protocol distributes randomly the sensor nodes in sensor area, and BS calculates distances between it and different nodes. The distance is calculated according of location information of each node.

b. Setup phase: in this stage, three different regions are organized, using the location information of SNs. This organization is to provide the same management for nodes belonging to the same region in the network. Nodes close to BS form the first region, where the direct communication is used without any clustering organization. The other two regions are structured in clusters.

c. Cluster head election: This operation concerns the two clustered regions, where a new concept of $\mathrm{CH}$ election is used. This, to improve LEACH limitations by considering the energy of nodes as the aim metric in $\mathrm{CH}$ election process and using threshold level energy Tc. The current $\mathrm{CH}$ of each cluster is changed once its energy is below Tc, else it continues its $\mathrm{CH}$ role. The election process is launched only in this cluster. The current $\mathrm{CH}$ is replaced only of the current $\mathrm{CH}$ achieves the Threshold energy, and the next $\mathrm{CH}$ must also have a higher energy level than the Threshold. If no cluster node has more energy than Tc, the next $\mathrm{CH}$ is randomly selected as the LEACH process.

d. Transmission: A TDMA schedule is created by each $\mathrm{CH}$ for its members. Each $\mathrm{CH}$ gathers data from all SNs in its cluster and transfers data to gateway node, according of its TDMA schedule created by the gateway node. The gateway transfers both $\mathrm{CHs}$ data to BS. Whereas, the data is transmitted directly to BS in first region.

\subsection{Clustering location based LEACH (CLOC-LEACH)}

In this section we discuss our contribution named CLOC-LEACH, which presents an improvement of LEACH protocol. We introduce three techniques to minimize energy of sensor node and prolong stability period of the network. CLOC-LEACH divides the network into four equal logical regions, and clustering scheme is adopted in each region. In addition, an optimal process of $\mathrm{CH}$ selection is implemented in each cluster, by using SN nodes the as principal metric to select a $\mathrm{CH}$. We deploy a gateway node, positioned in the center of sensing zone, to provide energy conservation and extend lifetime of WSN. The gateway is responsible to gather information from all $\mathrm{CHs}$, and transmits an aggregated packet to BS.

This contribution is proposed, to enhance some features like WSN lifetime, period of stability and clustering mechanism for an efficient WSN performance. Figure 2 shows topology of CLOC-LEACH protocol. CLOC-LEACH is divided in four steps:

a. Initialization phase: SNs are distributed with randomly manner in sensing zone, and BS sends a message hello to each node, and receives the location information as response from each sensor node. According of this information, the BS measures the distance between it and all SNs. Each SN uses its own data table, to keep: Its residual energy, its location in sensing zone and his distance from BS. A gateway node is located in the center of sensing zone.

b. Setup phase: The uniform organization of SNs in the sensing zone, provides more stability and energy conservation. In this step, we structure the sensing zone into four clusters, and each node is assigned to its appropriate cluster according of its location information. Nodes of the similar region of sensor area form the same cluster. CLOC-LEACH adopts this organization, to minimize the communication between nodes and more energy conservation. Each node communicates only with the nodes belonging to its cluster and avoid energy loss caused by transmission between distant nodes.

c. Cluster head election: This operation is executed in each cluster, independently of other clusters. CLOC$\mathrm{LEACH}$ performs the randomly election of $\mathrm{CH}$ used in LEACH. In this step, CLOC-LEACH adopts an optimal strategy, to select $\mathrm{CH}$ in each cluster unlike LEACH. We adopt the remaining energy of SN as the key metric to elect $\mathrm{CHs}$. In the first turn, all SNs have the same initial energy and the $\mathrm{CH}$ at each 
cluster is chosen randomly. We use a threshold of energy is to start the process of $\mathrm{CH}$ election. The current $\mathrm{CH}$ will remain in its leader role since its amount of energy is higher or equal to the threshold Tc of energy defined by the algorithm. When its remaining energy is below Tc, the process of $\mathrm{CH}$ election is launched only in the cluster concerned, and the node with the energy above or equal to the threshold will be elected new $\mathrm{CH}$. If all sensor nodes have the energy below the $\mathrm{Tc}$, the $\mathrm{CH}$ is chosen by using the selection process used in LEACH.

d. Transmission phase: A TDMA schedule is created by each $\mathrm{CH}$ for its cluster members and diffuses it for all nodes into its cluster. The $\mathrm{CH}$ of each cluster collects data from its cluster nodes and transfers an aggregate packet to the gateway respecting its TDMA schedule created by the gateway node. The communication inter-cluster is used through a gateway node, to offer a load balancing between nodes and avoid the loss of energy due to inter-cluster communication. The gateway gathers and fuses data from all $\mathrm{CHs}$ and sends them fused to the BS.

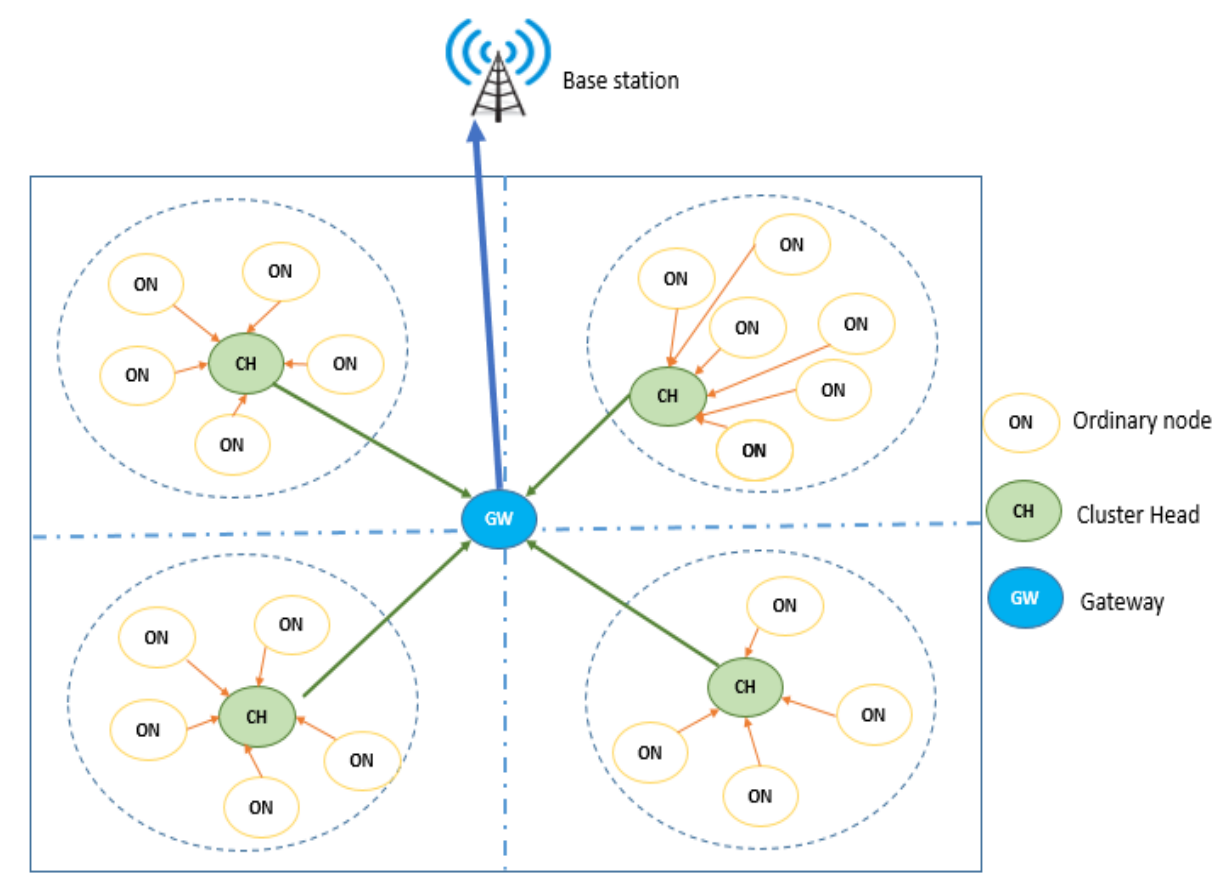

Figure 2. CLOC-LEACH architecture

\section{RESULTS AND ANALYSIS}

In this paragraph, we measure of CLOC-LEACH performances through matrix laboratory (MATLAB) simulator on the following metrics: repartition of dead nodes, number of alive nodes and energy consumed in WSN. We discuss simulations results of CLOC-LEACH protocol and compare them with LEACH protocol and our previous contribution LOC-LEACH protocol. The simulation is performed for 100 nodes distributed on sensor area of $100 \times 100 \mathrm{~m} 2$, the base station is positioned distant from the sensing zone at the position $(50,100)$ and considered stationary throughout of the simulation, the gateway node is positioned in the center of the sensing zone $(50,50)$ it is stationary after deployment. Table 1 shows the parameters used in our simulation.

Table 1. Simulation parameters

\begin{tabular}{cc}
\hline Parameter & Value \\
\hline Size of network & $100 * 100 \mathrm{~m}$ \\
Base station & 50,120 \\
Initial energy & $0.5 \mathrm{~J}$ \\
Size of packet & $4000 \mathrm{bits}$ \\
Energy cost of data aggregation & $5 \mathrm{pJ} / \mathrm{bit}$ \\
Total nodes & 100 \\
Efs & $10 \mathrm{pJ} / \mathrm{bit} / \mathrm{m} 2$ \\
Eelec & $5 \mathrm{~nJ} / \mathrm{bit}$ \\
Eamp & $0.0013 \mathrm{pJ} / \mathrm{bit} / \mathrm{m} 4$ \\
\hline
\end{tabular}




\subsection{Network stability}

The period of stability is considered as the main phase to extend in WSN, because it represents the period when the network works with all nodes before the first dead. CLOC-LEACH maintains the performance obtained by LOC-LEACH, and performs significantly LEACH results, due to the organization of nodes into clusters according of their location and using inter-communication throughout a getaway positioned in the middle of network. In Figure 3 shows that the number of alive nodes in CLOC-LEACH is higher than LEACH. The results obtained by CLOC-LEACH are significantly better than LEACH, in terms of stability period.

\subsection{Network lifetime}

CLOC-LEACH prolongs network lifetime better than the both protocols LEACH and LOC-LEACH, due to its strategy based in a uniform organization of nodes, and $\mathrm{CH}$ election process which takes into consideration, the amount energy of node and the distance between BS and different nodes. The Figure 4 shows that CLOC-LEACH increases the WSN lifetime than LEACH. This improvement, results from the optimized $\mathrm{CH}$ selection for each cluster independently of other clusters, which reduce the overhead due to the clustering operation. In addition, the use of a gateway node for inter-cluster communication reduces the additional tasks of $\mathrm{CHs}$ and provides best energy consumption. The Figure 5 represents the comparison between LEACH, LOC-LEACH and CLOC-LEACH.

\subsection{Energy consumption}

The Figure 6 shows that CLOC-LEACH allows more conservation of energy of the nodes. The various improvements added to $\mathrm{LEACH}$, provide more conservation of energy conservation of nodes, due to the optimal process of $\mathrm{CH}$ selection, and minimization of $\mathrm{CH}$ charges by using a gateway node for intercluster transmission.

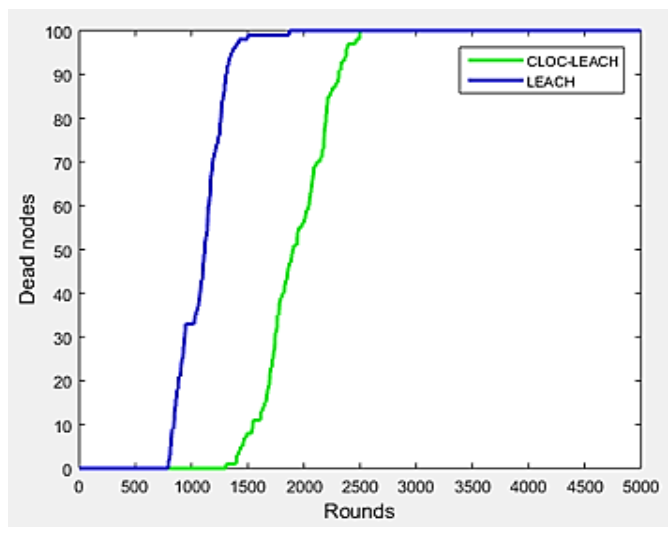

Figure 3. Comparison of network stability

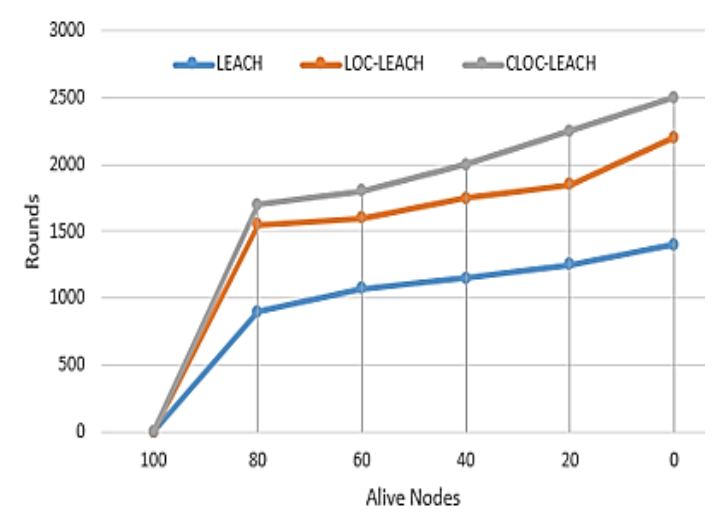

Figure 5. Comparison of alive nodes: LEACH, LOC-LEACH and CLOC-LEACH

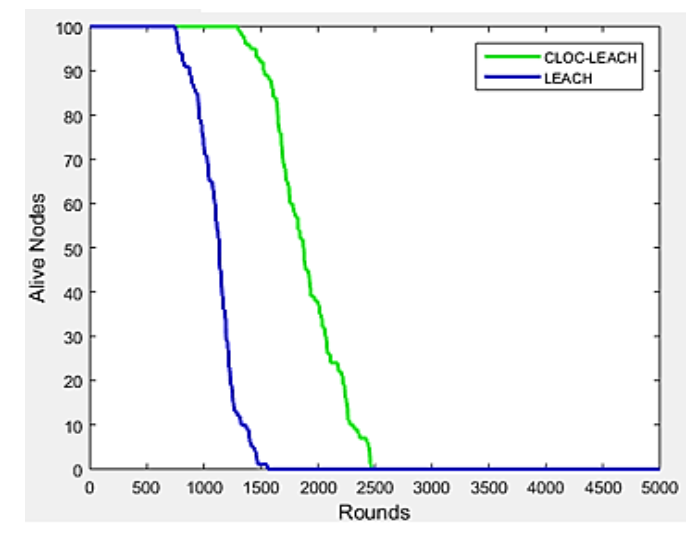

Figure 4. Comparison of alive nodes

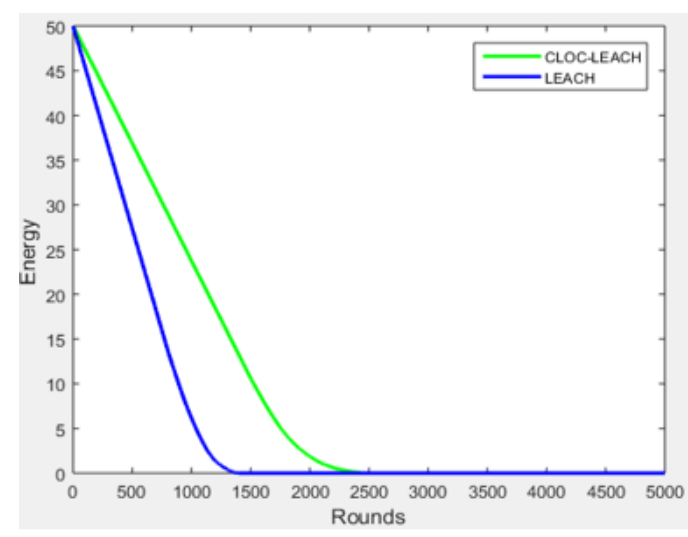

Figure 6. Comparison of energy consumption 


\section{CONCLUSION}

CLOC-LEACH protocol is presented in this work, to improve an existing protocol regarding period of stability and energy consumption in WSN. Load balancing between nodes in WSN and efficient organization of nodes in sensing area are the main objectives of our work. CLOC-LEACH divides the sensing zone into four clusters according of the location of sensor nodes. In addition of this organization in clusters, CLOC-LEACH adopts an efficient method of $\mathrm{CH}$ election, by using the amount energy of nodes as the key metric to select CHs. Results of simulation show that CLOC-LEACH improves considerably stability period. It prolongs WSN lifetime in comparison with LEACH and LOC-LEACH protocols due to the repartition of dead nodes and energy conservation. Thus, the proposed contribution allows better performances than LEACH protocol in the different presented cases. However, this contribution needs more improvements to reach robust, efficient, and scalable routing protocol for WSN. In our future steps, we are going to be interested in the intra-luster organization, to improve our results.

\section{REFERENCES}

[1] M. P. Đurišić, Z. Tafa, G. Dimić, and V. Milutinović, "A survey of military applications of wireless sensor networks," Mediterranean Conference on Embedded Computing MECO, pp. 196-199, 2012.

[2] T. Jamal and S. A. Butt, "Low-energy adaptive clustering hierarchy (LEACH) enhancement for military security operations," Journa of Basic Applied Science Research, vol. 7, no. 5, pp. 12-17, 2017.

[3] N. S. Ali, Z. A. A. Alyasseri, and A. Abdulmohson, "Real-time heart pulse monitoring technique using wireless sensor network and mobile application," International Journal of Electrical and Computer Engineering (IJECE), vol. 8, no. 6, pp. 5118-5126, 2018, doi: 10.11591/ijece.v8i6.pp5118-5126.

[4] H. H. Qasim, A. E. Hamza, L. Audah, H. H. Ibrahim, H. A. Saeed, and M. I. Hamzah, "Design and implementation home security system and monitoring by using wireless sensor networks WSN/internet of things IoT," International Journal of Electrical and Computer Engineering (IJECE), vol. 10, no. 3, pp. 2617-2624, 2020, doi: 10.11591/ijece.v10i3.pp2617-2624.

[5] H. Sayuti et al., "Smart home and ambient assisted living based on the internet of things," International Journal of Electrical and Computer Engineering (IJECE), vol. 7, no. 3, pp. 1480-1488, 2017, doi: 10.11591/ijece.v7i3.pp1480-1488.

[6] A. Rodríguez, C. Del-Valle-Sot, and R. Velázquez, "Energy-efficient clustering routing protocol for wireless sensor networks based on yellow saddle goatfish algorithm," Mathematics, vol. 8, no. 9, 2020, doi: 10.3390/math8091515.

[7] S. A. Kumar, P. Ilango, and G. H.Dinesh, "A modified LEACH protocol for increasing lifetime of the wireless sensor network," Cybernetics and Information Technologies, vol. 16, 2016, doi: 10.1515/cait-2016-0040.

[8] A. S. Al-Zubaidi, A. A. Ariffin, and A. K. Al-Qadhi, "Enhancing the stability of the improved-LEACH routing protocol for WSNs," Journal of ICT Research and Application, vol. 12, no. 1, 2018, doi: 10.5614/itbj.ict.res.appl.2018.12.1.1.

[9] S. K. Singh, P. Kumar, and J. P. Singh, "A survey on successors of LEACH protocol," IEEE Access, vol. 5, pp. 4298-4328, 2017, doi: 10.1109/ACCESS.2017.2666082.

[10] G. S. Sara and D. Sridharan, "Routing in mobile wireless sensor network : A survey," Telecommun Systems, vol. 57, pp. 51-79, 2013.

[11] G. Anastasi, M. Conti, M. D. Francesco, and A. Passarella, "Energy conservation in wireless sensor networks : A survey," Ad Hoc Networks, vol. 7, no. 3, pp. 537-568, 2009, doi: 10.1016/j.adhoc.2008.06.003.

[12] S. Rani, J. Malhotra, and R. Talwar, "Energy efficient chain based cooperative routing protocol for WSN," Applied Soft Computing ,vol. 35, pp. 386-397, 2015, doi: 10.1016/j.asoc.2015.06.034.

[13] W. R. Heinzelman, A. Chandrakasan, and H. Balakrishnan, "Energy-efficient communication protocol for wireless microsensor networks," Proc. of the Hawaii International Conference on System Sciences, vol. 2, 2000, doi: 10.1109/HICSS.2000.926982.

[14] M. R. E. Ouadi and A. Hasbi, "LOC-LEACH: An enhanced LEACH protocol for WSNs," International Journal of Advanced Trends in Computer Science and Engineering, vol. 9, no. 4, pp. 4567-4574, 2020, doi: 10.30534/ijatcse/2020/55942020.

[15] W. B. Heinzelman, A. P. Chandrakasan, and H. Balakrishnan," An application-specific protocol architecture for wireless microsensor networks," IEEE Transactions on Wireless Communications, vol. 1, no. 4, pp. 660-670, 2002, doi: 10.1109/TWC.2002.804190.

[16] V. Loscri, G. Morabito, and S. Marano, "A two-levels hierarchy for low-energy adaptive clustering hierarchy (TL-LEACH)," VTC-2005-Fall. 2005 IEEE 62nd Vehicular Technology Conference, 2005, pp. 1809-1813, doi: 10.1109/VETECF.2005.1558418.

[17] M. B. Yassein, A. Al-zou'bi, Y. Khamayseh, and W. Mardin, "Improvement on LEACH protocol of wireless sensor network (VLEACH)," Int. J. of Digital Content Technology and its Applications, 2009, doi: 10.1109/SENSORCOMM.2007.4394931.

[18] F. Xiangning and S.Yulin, "Improvement on LEACH protocol of wireless sensor network," International Conference on Sensor Technologies and Applications, 2007, pp. 260-264, doi: 10.1109/SENSORCOMM.2007.4394931.

[19] L. T. Nguyen, X. Defago, R. Beuran, and Y. Shinoda, "An energy efficient routing scheme for mobile wireless sensor networks," IEEE International Symposium on Wireless Communication Systems, 2008, pp. 568-572, doi: 10.1109/ISWCS.2008.4726120.

[20] S. K. Sing, M. P. Singh, and D. K. Singh, "A survey of energy-efficient hierarchical cluster-based routing in wireless sensor networks," International Journal of Advanced Networking and Applications, vol. 2, no. 2, pp. 570-580, 2010.

[21] J. Liu and C. V. Ravishankar, "LEACH-GA: Genetic algorithm-based energy-efficient adaptive clustering protocol for wireless sensor networks," International Journal of Machine Learning and Computing, vol. 1, no. 1, 2011.

[22] P. Sivakumara and M. Radhika, "Performance analysis of LEACH-GA over LEACH and LEACH-C in WSN," Procedia Computer Science, vol. 125, pp. 248-256, 2017, doi: 10.1016/j.procs.2017.12.034.

[23] N. Sharma and V. Verma, "Energy efficient LEACH protocol for wireless sensor network," IJINS, vol. 2, no. 4, pp. 1-11, 2013.

[24] R. E. Mohamed; A. I. Saleh, M. Abdelrazzak, and A. S. Samra, "Survey on wireless sensor network applications and energy efficient routing protocols," Wireless Personal Commun, vol. 101, pp. 1019-1055, 2018, doi: 10.1007/s11277-018-5747-9.

[25] M. R. El Ouadi and A. Hasbi, "Comparison of LEACH and PEGASIS hierarchical routing protocols in WSN," International Journal of Online and Biomedical Engineering (iJOE), vol. 16, no. 9, 2020 